\title{
Diagnosis of von Hippel-Lindau Syndrome Following Endoscopic Ultrasound of the Pancreas
}

\author{
1Jennifer Cannon, ${ }^{2}$ Paul G Gauger, ${ }^{3}$ Gerard M Doherty, ${ }^{4}$ James M Scheiman \\ ${ }^{1}$ Fellow in Endocrine Surgery, DeWitt Daughtry Family, Department of Surgery, University of Michigan Medical School, Ann \\ Arbor, MI-48109 \\ ${ }^{2}$ Associate Professor, Division of Endocrine Surgery, University of Michigan Medical School, Ann Arbor, MI-48109 \\ ${ }^{3}$ Professor, Department of Surgery, Division of Endocrine Surgery, University of Michigan Medical School, Ann Arbor, MI-48109 \\ ${ }^{4}$ Professor, Department of Internal Medicine, Gastroenterology, University of Michigan Medical School, Ann Arbor, MI-48109 \\ Correspondence: Paul G Gauger, William J Fry Professor of Surgery, Associate Professor of Surgery and Medical Education \\ University of Michigan, Department of Surgery, 1500 East Medical Center Drive, Taubman Center-TC2920D, Ann Arbor \\ Ml-48109-0331, Phone: 734-936-0231, Fax: 734-936-5830, e-mail: pgauger@umich.edu
}

\begin{abstract}
Objective: To report a case of von Hippel-Lindau (VHL) syndrome diagnosed by endoscopic ultrasound (EUS) of the pancreas in a patient with no family history of the disease and without any other clinical manifestations of VHL.

Methods: We describe the clinical presentation of the case and discuss its management.

Results: A 33-year-old woman was evaluated by EUS for possible pancreatic carcinoma. Based upon the sonographic appearance of her pancreas, VHL was suggested as a diagnosis and subsequently confirmed by genetic testing. The pancreatic lesions were treated surgically and pathology revealed pancreatic islet cell tumors.

Conclusion: This is the first report of a patient in which the first suggestion of a VHL diagnosis was made on EUS appearance of pancreatic lesions alone.
\end{abstract}

Keywords: Von Hippel-Lindau syndrome, endoscopic ultrasound, pancreatic neoplasm.

\section{CASE REPORT}

A 33-year-old woman complained of abdominal pain, nausea, vomiting, and a 10-lb weight loss. A CT scan of the abdomen revealed a pancreatic mass. An ERCP was performed and was unremarkable but limited by the fact that the operator was unable to cannulate the pancreatic duct. Because of the unresolved concern for pancreatic carcinoma, she was referred for endoscopic ultrasound. Past medical history included childhood meningitis with resultant epilepsy. Neither her parents nor any of her 10 siblings had renal stones or tumors, pituitary tumors, pancreatic tumors, pheochromocytomas, or other lesions suggestive of an inherited endocrinopathy.

Endoscopic ultrasound revealed multiple pancreatic lesions. A $1.5 \mathrm{~cm} \times 1.5 \mathrm{~cm}$ solid, smooth, hypoechoic mass was noted in the head. Multiple cystic pancreatic masses up to $1.3 \mathrm{~cm}$ with multiple septae were noted throughout the pancreatic neck, body, and tail (indicated by white arrows in Figs 1 and 2). The constellation of pancreatic abnormalities was concerning for a syndrome such as von Hippel-Lindau, with MEN 1 also a possibility.

On physical exam, she was a thin female with normal vital signs. No skin lesions were present. Her neurological exam was normal. Cardiovascular and pulmonary exams were normal. Her

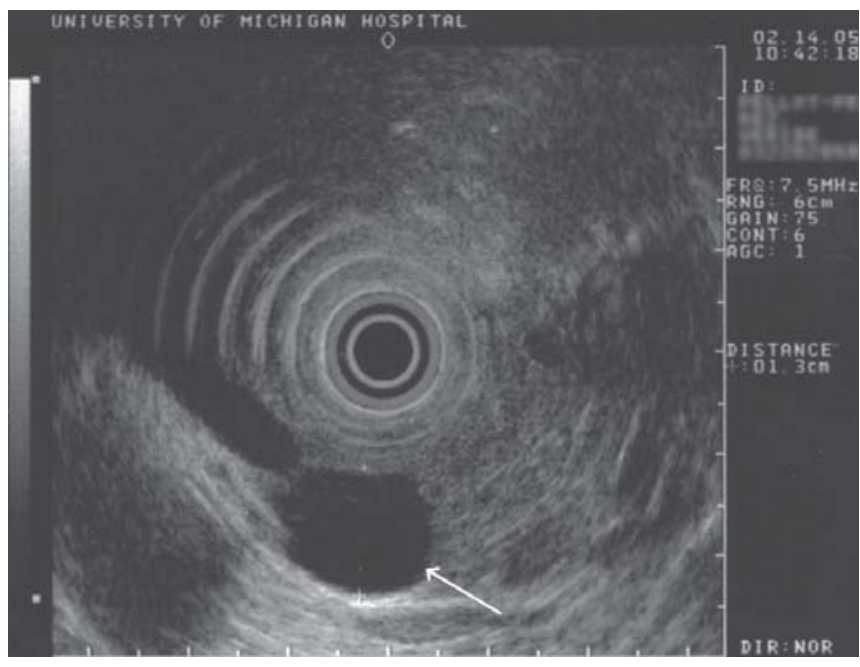

Fig. 1: Cystic pancreatic mass

abdomen was soft, tender to palpation in the midepigastrium and right upper quadrant, but without masses or organomegaly.

A repeat IV-contrast enhanced spiral CT scan of the abdomen (Fig. 3) redemonstrated the presence of pancreatic masses: at least two hyperattenuating masses were seen in the uncinate process and head of the pancreas and at least two 


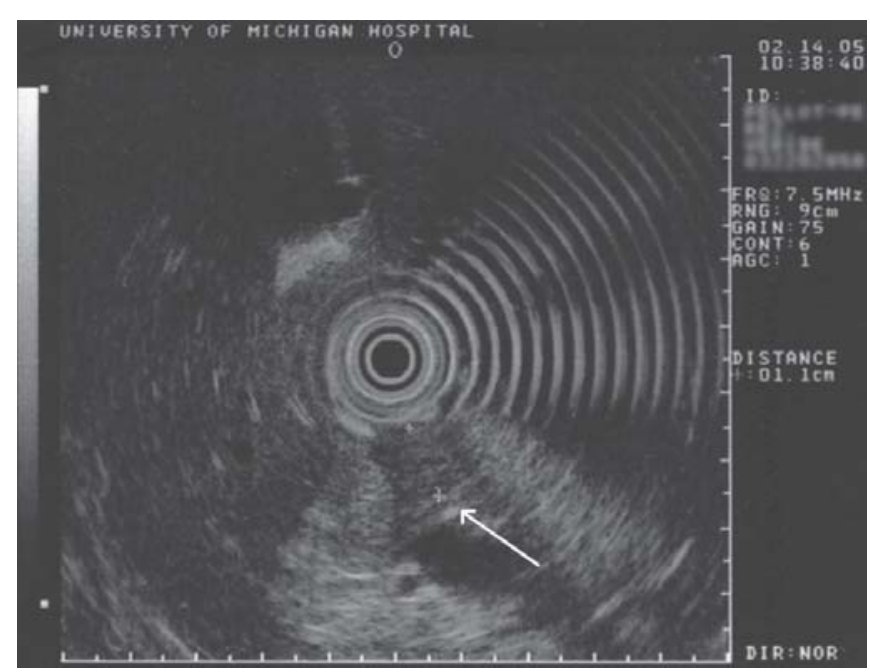

Fig. 2: Solid pancreatic mass

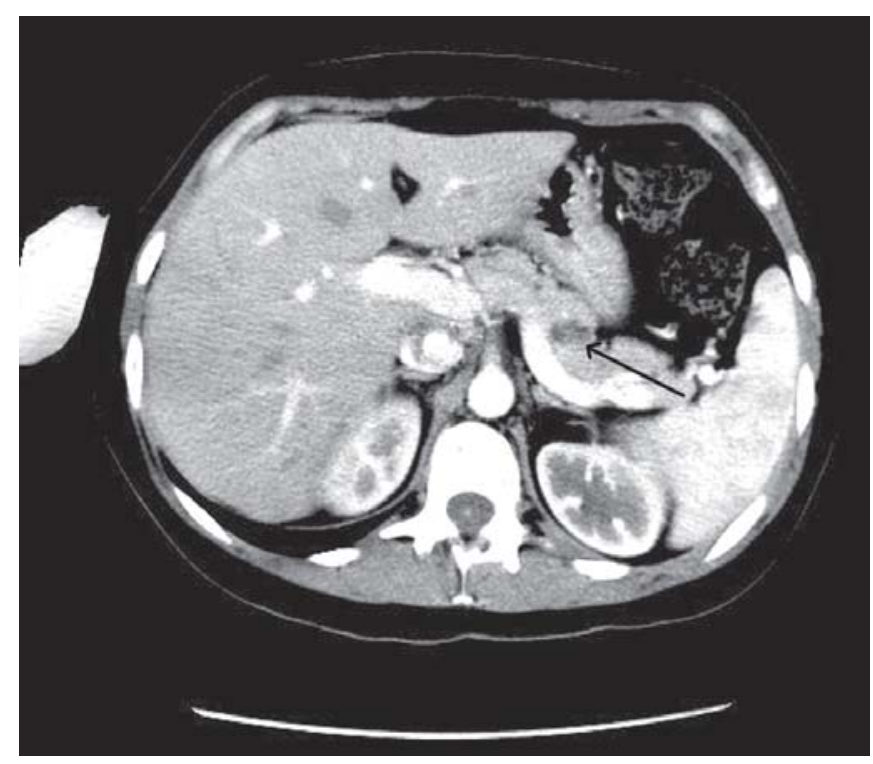

Fig. 3: Cystic pancreatic mass, redemonstrated on CT scan

hypoattenuating masses were visualized in the body and tail. The kidneys, adrenals, and other organs were all normal in appearance. Plasma free normetanephrine level was negligibly elevated at $0.93 \mathrm{nmol} / \mathrm{L}$ (normal < $0.89 \mathrm{nmol} / \mathrm{L}$ ). Parathyroid hormone, gastrin, and chromogranin a levels were normal. Genetic analysis was obtained to investigate the possibility of von Hippel-Lindau syndrome. The patient was heterozygous for the A to G mutation at nucleotide 446 of the VHL gene that changed a codon for asparagine (ATT) to one for serine (AGT) at amino acid position 78.

Given the solid appearance of the tumors in the head and uncinate process, as well as her symptoms of abdominal pain, the patient underwent exploratory laparotomy with intraoperative ultrasound and enucleation of a total of three pancreatic masses. Her postoperative course was complicated by a urinary tract infection and a segmental pulmonary embolus. Final pathology from all three masses demonstrated noninvasive pancreatic islet cell tumors.

\section{DISCUSSION}

von Hippel-Lindau syndrome is a highly penetrant autosomal dominant disorder traced to a mutation at chromosome 3p2526 . It has a birth incidence of approximately $1 / 36,000$, with nearly $20 \%$ of cases arising from de novo mutations, without a family history. ${ }^{1-3}$ It causes a variety of neoplastic manifestations, including retinal and central nervous system hemangioblastomas, renal cell carcinomas, pheochromocytomas, epididymal cysts, and pancreatic lesions. ${ }^{4,5}$ Pancreatic cysts account for the majority of these, on the order of 55-67\%, with approximately $15 \%$ representing islet cell tumors, and the remainder composed of benign serous cystadenomas, hemangioblastomas, adenocarcinomas, renal cell cancer metastases, and indeterminate pancreatic masses or a combination of these lesions. ${ }^{4}$

The majority of VHL patients are diagnosed after the discovery of central nervous system tumors or after affected family members prompt genetic screening. Up to $12 \%$ of VHL patients may have pancreatic lesions as the only abdominal manifestation, ${ }^{6}$ as was the case with this patient. They may precede development of other manifestations of the disease by several years. ${ }^{6}$ Pancreatic lesions are seldom symptomatic and are generally detected incidentally on routine abdominal surveillance, usually computed tomography (CT) or standard abdominal ultrasound (US). Endoscopic ultrasound (EUS) is frequently employed to better characterize pancreatic lesions and may be used to obtain biopsy specimens of suspected malignant tumors, but rarely does it suggest a diagnosis, exclusive of other imaging, as in our patient's case. A previous case report describing the use of EUS in a patient with other clinical manifestations of VHL has been published. ${ }^{7}$ This is the first report of a patient in which the first suggestion of this diagnosis was made on EUS appearance of pancreatic lesions alone.

\section{REFERENCES}

1. Woodward ER, ME von Hippel-Lindau disease and endocrine tumour susceptibility. Endocr Relat Cancer 2006;13:415-25.

2. Maher ER, IL Yates JR, et al. von Hippel-Lindau disease: A genetic study. J Med Genet 1991;28:443-47.

3. Richards RM, PS, Zbar B, et al. Molecular analysis of de novo germline mutations in the von Hippel-Lindau disease gene. Hum Mol Gen 1995;4:2139-43.

4. Hammel PR, VV, Terris B, et al. Pancreatic involvement in von Hippel-Lindau disease. Gastroenterology 2000;119:1087-95.

5. Ling H, CM, Shaefer O, et al. When to look for von HippelLindau disease in gastroenteropancreatic neuroendocrine tumors? Neuroendocrinology 2004;80(suppl 1):39-46.

6. Yadav D, HJ, Pitchumoni CS. Image of the Month: von HippelLindau disease. Gastroenterology 2001;120:788-1058.

7. Elli L, BE, Portugalli V, et al. Pancreatic involvement in von Hippel-Lindau disease: Report of two cases and review of the literature. Am J Gastroenterol 2006;101:2655-58. 